\title{
Germanica
}

\section{Franz Fühmann et le Conte}

Franz Fühmann und das Märchen

\section{Françoise Barthélémy-Toraille}

\section{(2) OpenEdition}

Journals

Édition électronique

URL : http://journals.openedition.org/germanica/1359

DOI : 10.4000/germanica.1359

ISSN : 2107-0784

Éditeur

Université de Lille

Édition imprimée

Date de publication : 1 janvier 1992

ISSN : 0984-2632

Référence électronique

Françoise Barthélémy-Toraille, « Franz Fühmann et le Conte », Germanica [En ligne], 11 | 1992, mis en ligne le 07 février 2014, consulté le 10 décembre 2020. URL : http://journals.openedition.org/ germanica/1359; DOI : https://doi.org/10.4000/germanica.1359

Ce document a été généré automatiquement le 10 décembre 2020.

(c) Tous droits réservés 


\title{
Franz Fühmann et le Conte
}

\author{
Franz Fühmann und das Märchen
}

Françoise Barthélémy-Toraille

Et les spectateurs écoutaient le conte et
l'entendaient murmurer : c'est de toi qu'il est ici
question.
Franz Fühmann
Vingt-deux jours ou la moitié de la vie ${ }^{1}$

Écrivain venu à la prose après avoir débuté comme poète, Franz Fühmann s'impose à ses lecteurs à la fois comme narrateur et comme penseur. Le conte est à la source de son écriture, il en constitue un des aspects: source si l'on envisage son activité de réécriture de contes et mythes appartenant au fonds culturel universel, aspect de son écriture lorsque l'on considère certaines de ses œuvres selon les catégories du conte. À cela, il convient d'ajouter que si Fühmann, qui est un grand auteur de livres pour enfants, reprend pour eux des grands thèmes de la littérature universelle, il est aussi «l'inventeur » de contes modernes écrits à leur intention. Il convient enfin de noter que la réflexion sur le conte, sur sa relation au mythe, sur sa fonction, tiennent une place importante dans l'œuvre de Fühmann, en particulier dans Vingt-deux jours ou la moitié de la vie ainsi que dans les Essais ${ }^{2}$.

2 Dans l'un de ses Essais intitulé «La part du mythe dans la littérature », Fühmann pose les bases de sa conception du conte par rapport au mythe. Par conte, comme nous le verrons par la suite, Fühmann entend ici « conte populaire ». Le conte se situe pour lui dans un monde intact, ou Bien et Mal, Bon et Mauvais s'opposent de manière indiscutable, dans une lutte qui aboutit à un triomphe sans partage du Bien sur le Mal :

On peut complètement et sans difficulté intégrer le conte dans un système de coordonnées morales... À chacune des questions que soulève un conte on peut répondre clairement par oui ou par non ${ }^{3}$.

3 Le Prince est bon, le Sorcier méchant, et le conte connaît une issue heureuse par le triomphe du Prince sur le principe du Mal, dans la négation de la contradiction qui les oppose. Dans le mythe au contraire, Zeus unit les deux principes du Bien et du Mal, il unit en lui-même la contradiction qu'il s'agit de surmonter, et le mythe n'aboutit pas, 
en son ultime péripétie, à un happy end inéluctable. On peut, nous dit Fühmann, voir dans les contes « des mythes déchus ", et aussi

une des métamorphoses derrière lesquelles sommeillent les mythes anciens, et il est possible de réveiller les dormeurs, comme la Belle au Bois Dormant dans son château qu'entourent les ronces ${ }^{4}$.

Il faut dépasser le happy-end, et retrouver dans les contes les contradictions cachées dont ils sont porteurs, car la contradiction, dans son aspect dialectique, est créatrice et féconde.

Cette réflexion sur le conte permet à Fühmann de dénoncer la littérature manichéenne qui marque, sous l'égide du réalisme socialiste, la littérature de R.D.A. à ses débuts :

Allons un pas plus loin et faisons une hypothèse de travail. Essayons d'imaginer une littérature qui a ceci de commun avec le conte qu'elle élimine elle aussi les contradictions de l'existence, mais se distingue par ailleurs du conte dans le fait qu'elle évite tout recours au surnaturel ou à ce qui ne serait pas naturel... Nous nous représentons donc une littérature sans contradictions internes, qui distingue bien nettement dans leur opposition le bien du mal, le vrai du faux, l'utile du nuisible, en $\mathrm{y}$ ajoutant non pas des lutins et des sorcières, pas de Tom Pouce ni de mangeur d'hommes et pas davantage de rois et de princes et de pauvres charbonniers, mais des personnages de notre vie de tous les jours : des chefs de brigades, des paysans d'une coopérative, des représentants du Parti, des soldats de l'armée du peuple, des jeunes pionniers et des maîtres méritants, et tout ceux qu'on voudra - une telle littérature est tout à fait imaginable ${ }^{5}$,

mais, ajoute Fühmann,

je tiens Zeus et Léda pour infiniment plus proches de l'homme que maint petit

couple de poèmes ou de récits contemporains ${ }^{6}$,

personnages de la réalité quotidienne tels qu'on les rencontre dans certains des fleurons de la littérature écrite sous l'égide du « réalisme socialiste ». Le conte, qui voit triompher le Bien sur le Mal, est, tout comme cette littérature-là, en accord avec la morale en vigueur. Il n'est pas, lui non plus, dépourvu d'une certaine tendance didactique et moralisatrice. Au conte populaire, Fühmann oppose le « Kunstmärchen »", à la fois œuvre d'art et création de l'artiste, ou même artifice, qui accorde aux contradictions propres à la vie, donc à la dialectique, la place que son auteur aura décidé de lui attribuer. Cela le rapproche et de la vie, et du mythe.

5 Fühmann ne se contente pas d'une réflexion théorique sur le conte et sur ses rapports au mythe, mais il met aussi cette réflexion en pratique en écrivant lui-même des contes : après une période où il reprend et réécrit des motifs et sujets de la littérature universelle, Reineke Fuchs, Shakespeare, Homère etc. ${ }^{8}$, Fühmann laisse libre cours à son imagination créatrice et « invente » des contes pour ses jeunes lecteurs. Il est d'autre part possible de mettre en évidence la présence, dans certains récits fühmanniens, d'éléments propres au conte.

6 Franz Fühmann se livre, dans le cas de « Reineke Fuchs », du « Niebelungenlied ou d'un certain nombre de grands mythes de l'Antiquité, à une "réécriture " guidée sans cesse par la question qu'il pose dans une postface à «Reineke Fuchs" rédigée en 1981 à propos de ce texte écrit en 1961, «Qu'est-ce que cela nous apprend? »9. Par cette question, l'auteur souligne l'actualité pour ses lecteurs d'un certain nombre de problèmes - relations sociales, comportements... - mis en lumière dans ces récits.

7 Une évolution du rapport de Fühmann à son modèle est déjà sensible avec les «Contes d'après Shakespeare » : un narrateur, un conteur plutôt, s'adresse à de jeunes lecteurs, ses contemporains, dont l'imagination est prête en quelque sorte à se laisser prendre 
par la main. Le conteur interpelle ses lecteurs au détour du récit, et les fait ainsi « entrer dans le conte»:

Or vivaient à Athènes cinq personnages qui n'avaient pas le cœur à la plaisanterie... Et pourquoi ces cinq-là n'étaient-ils pas de joyeuse humeur? Eh bien, mes enfants, c'est une longue histoire, touffue et confuse, et en plus emmêlée et embrouillée! Soyez très attentifs, si vous voulez la comprendre comme il faut. Alors, pointez vos oreilles comme un caniche, et écoutez bien ${ }^{10}$.

De telles remarques, qui visent à renforcer l'attente, mais aussi l'attention de ses jeunes lecteurs, ont la même fonction que les "questions rhétoriques" qui, tout en ralentissant le flux d'un récit linéaire, créent des ruptures de ton, et en fait évitent l'écueil de la monotonie. Le jeu des questions et réponses contribue lui aussi à «intégrer» les jeunes lecteurs dans l'action: "Que pouvait-il alors advenir? Exactement ce à quoi vous pensez... ${ }^{11}$ Lorsque les personnages se retrouvent dans la forêt ténébreuse, l'auteur quitte en quelque sorte le fil du récit pour mieux créer «l'illusion du conte»;

9 Les voici donc tous les quatre dans le bois qui entoure la ville - et nous autres? Les y suivons-nous ? Souvenez-vous, il fait nuit, la forêt est effrayante la nuit, les chouettes hululent et les hiboux font kiwitt kiwitt, les chauves-souris volètent avec leurs ailes nues et des feux follets bleus se balancent et s'esbaudissent à travers la sapineraie crépusculaire! Des yeux de lynx brillent, des yeux de renard étincellent; le chat sauvage aiguise ses griffes contre l'écorce du chêne et de noirs sangliers armés de défenses trottinent dans la combe aux bouleaux... Il fait nuit, sou venez-vous, vous n'y voyez goutte... Il ne fait pas bon être cette nuit dans la forêt, y suivrons-nous cependant nos amoureux ? Nous y risquerons-nous tout de même?

Alors venez, donnez-moi la main, et soyez sans peur, quoi qu'il advienne... ${ }^{12}$.

Dans un tel passage, tout en faisant la preuve de sa maîtrise de Fart de capter l'attention de ses lecteurs - de son public -, Fühmann laisse libre cours à sa fantaisie d'écrivain pour qui le langage est matière première qui permet jeux d'assonances, d'allitérations, de rimes et de répétition. La jubilation du créateur est perceptible, pardelà même le récit et son objet. Le langage devient lui-même matériau mais aussi objet de l'écriture ${ }^{13}$. Le conte s'achève par un retour au réel, qui contribue à ancrer le merveilleux du conte dans la réalité quotidienne, à rendre floues les frontières entre le monde du conte et celui du réel :

Obéron et Titania... se sont fait la guerre pendant sept ans, ils veulent maintenant fêter comme il convient leur réconciliation!

Mais quand le temps semble à nouveau complètement ensorcelé, quand il gèle en été et que la neige fond en hiver, quand soudain le brouillard s'abat au beau milieu de jours lumineux, ou que sécheresse et déluges de pluie se succèdent, alors, vous savez ce qui se passe: la guerre a repris entre les deux elfes! Et quand alors le temps soudain s'apaise, que l'air se met à bruire et à vibrer comme sous le vol d'invisibles compagnies d'hirondelles, gardez-vous bien d'aller dans les bois! Peutêtre un nouveau songe d'une nuit d'été est-il en train de commencer tout près de vous $^{14}$.

11 Le conteur Fühmann se manifeste de manière plus lumineuse encore dans les récits dont la paternité lui revient complètement. Ces contes, dont certains sont rassemblés sous le titre de "Contes sur commande ", ont en général un destinataire ou dédicataire bien précis, sa fille, sa petite-fille, des enfants de son entourage, une jeune femme handicapée mentale, les élèves d'une école qu'orne, près de Stuttgart, une fresque de 
HAP Grieshaber, son ami. Là aussi, Fühmann s'adresse à son, ses lecteurs comme s'ils étaient présents et l'écoutaient.

«Les meilleurs lecteurs sont les enfants », disait Fühmann. Le conte Fühmannien mêle les éléments du réel quotidien de ses lecteurs et les éléments merveilleux : dans une ville qui a tout pour être réelle, et même un nom, Kötzschenbroda, avec un maire, une école et une maîtresse d'école, et des écoliers, des maisons et leurs fenêtres, habite une bête étrange qui pense " en triangle » (ne parle-t-on pas d'idées bien carrées, pourquoi n'y aurait-il pas d'idées triangulaires ?) et qui, son nom sans doute l'indique, prend plaisir à résoudre des énigmes ${ }^{15}$. Douée d'un épouvantable esprit de contradiction, elle se met en colère si on lui souhaite une excellente journée, car ce qu'elle veut, c'est une horrible, épouvantable journée qui lui laisse tout loisir d'être d'une humeur massacrante. Mais si c'est justement ce qu'on lui souhaite, elle s'en offusque :

Comment savez-vous donc que je veux avoir une horrible épouvantable journée ${ }^{16}$ Et l'interlocuteur reste sans voix. C'est là ce que retiennent les enfants, compagnons de jeux de cette bête étrange quand elle est d'humeur joyeuse: quand leur maîtresse affirme, péremptoire : trois fois trois neuf !, ils lui demandent: "Comment le savezvous? ». Par ce jeux de questionnement, dit le narrateur « les enfants apprirent à poser les bonnes questions ", "à poser des questions de manière astucieuse ${ }^{17}$. Et c'est bien cela qui importe. En mêlant situations qui relèvent du conte et éléments de leur quotidien - et tout particulièrement l'école, la relation enseignant-élève, mais aussi la notion même de relation hiérarchique ${ }^{18}$, Fühmann éclaire d'un jour nouveau une situation ordinaire: un animal fantastique tel que cette bestiole revêche ne se rencontre certes pas tous les jours, mais les enfants ne sont pas davantage habitués à remettre en question le savoir qui leur est transmis, ils ne sont pas éduqués à interroger jusqu'au moment où la réponse les satisfait. D'autre part, de même que la formule vidée de son sens par laquelle on se souhaite bonne journée est ici "inversée ", c'est la relation entre les enfants et leur maîtresse qui l'est aussi : la maîtresse se casse la tête pour résoudre le problème que lui posent les enfants, et apprend elle aussi le jeu $\mathrm{du}$ questionnement: les adultes peuvent à leur tour tirer des enseignements des questions que posent les enfants, c'est une des autres réflexions que peut inspirer ce petit texte.

Fühmann procède de manière analogue dans l'histoire de la petite fée qui savait cracher le feu ${ }^{19}$, ou dans celle d' ' Anna la petite sorcière, qu'on appelait Cloche-patte $»^{20}$ parcequ'elle avait une jambe plus courte que l'autre: Anna est différente, les autres petits sorciers et sorcières se moquent d'elle, mais elle leur montre que, grâce à ses deux jambes d'inégale longueur, elle possède des qualités qu'ils n'ont pas : celui qui est autre n'est pas inférieur, il lui faut cependant découvrir lui-même les qualités qui lui sont propres, tel est ici le «message ».

Dans l'univers des contes Fühmanniens, on retrouve, mais comme un clin d'œil, des éléments du conte populaire, dans la formulation - " Il était une fois », le recours aux répétitions, aux phrases-tiroir avec énumération d'une sorte de chaîne d'éléments qui successivement se déterminent ${ }^{21}$ - et dans les acteurs du conte: petites fées ou sorcières, animaux fantastiques doués de la parole, dragons et géants. Mais l'apport proprement fühmannien se situe à la fois dans ce jeu de va-et-vient entre l'univers de ceux auxquels il s'adresse et l'univers du conte, et dans une utilisation spécifique du langage considéré comme matériau. Le jeu sur le langage, sur les expressions "prises au pied de la lettre ", devient un des éléments du conte : les sorciers et sorcières vivent 
dans un monde qui est «l'inverse » du nôtre, il est alors logique que leur enfants aillent à l'école la nuit, et qu'ils dorment quand il fait jour, "qu'il règne dans la forêt une clarté qui vous donne la chair de poule, que des anneaux de soleil tachètent le sol couvert d'aiguilles et que le merle siffle d'une manière si étrange $»^{22}$. Les sorciers organisent une grande fête sportive à la première nouvelle lune qui suit la nuit de Walpurgis, avec des disciplines bien particulière: "courses de boucs" (mais «Bocksreiten » a en allemand une ambiguïté intraduisible !), « vol de manches à balais, saut à travers feu d'enfer authentique ». S'il est question de marcher sur les mains, tout est mis sens dessus dessous: Anna Cloche-patte n'a qu'à marcher sur les mains pour accéder à un monde à l'envers, où le maître assis à un banc, se fait interroger par les élèves, tous debout sur l'estrade. Et c'est justement parce qu'il est interdit aux petits sorciers d'aller « au bout du monde » qu'Anna, à force de marcher sur les mains et de voir les choses sens dessus dessous, ne souhaite rien plus ardemment que d'aller au bout du monde, et y parvient. Et en revient. Le thème de la transgression du tabou est appliqué ici à un sujet particulièrement sensible, celui du voyage et de la frontière infranchissable. (Le conte en question, publié pour la première fois en 1981 est l'un des "Contes sur commande » dont il est dit dans la préface à l'édition qu'ils ont été écrits peu de temps auparavant). La petite sorcière, une fois parvenue au bout du monde, qui en fait n'est qu'à "sept sauts de lièvre » de chez elle, derrière un brasier redoutable, voit une immense forêt, et deux géants en train de se battre. D'une expression du langage courant - au bout du monde - qui marque l'éloignement, l'inaccessible, Fühmann a fait une réalité concrète, a montré la transgression du tabou. Le langage, lorsqu'il est pris au pied de la lettre, devient un des éléments de dépaysement auxquels recourt le conte fühmannien, mais aussi un des acteurs du conte.

Cela devient encore plus évident dans cet ouvrage intraduisible qui s'intitule, reprenant la phrase par laquelle Hérodote ouvrit la joute poétique qui, selon le principe du «cadavre exquis » cher aux surréalistes, l'opposa à Homère "Die dampfenden Hälse der Pferde im Turm von Babel $»^{23}$. S'y rejoignent tous les éléments du «conte Fühmannien » : Des personnages qui peuvent servir de « modèles d'identification » aux lecteurs auxquels il s'adresse : des enfants, rassemblés dans une auberge de montagne, pendant les vacances, et par temps de pluie, donc doublement désœuvrés, tandis que les adultes "tuent le temps " à leur manière ; et deux personnages fantastiques, sortis semble-t-il d'un scintillement plus intense de la lampe verte du poste de radio: Schopenhauer, que les enfants surnomment affectueusement Schopi, et une espèce de petit fakir enturbanné qui s'avère être le grand maître des jeux du langage, Küslübürtün. Tout en racontant des histoires aux enfants, donc aux lecteurs, ces deux personnages, et avec eux Fühmann, font découvrir les facettes et les richesses du langage : palindromes de plus en plus longs, mots qui se lisent dans les deux sens en changeant de signification, anagrammes, homonymes, phrases ou mots composés qui n'utilisent qu'une seule voyelle (ce qui permet à Fühmann de raconter l'histoire - là encore intraduisible - d'une petite fée qui a mal à son orteil, et d'aboutir au mot possible - "Schneeseekleerehfeedrehzehweh »... mots qui déplacent leurs syllabes ou se rajoutent des lettres, comme dans l'histoire du petit « und » qui pleure d'être seul au monde, mais ne veut pas qu'on lui ajoute en guise de tête un «H », ni un « $M$ », ni un « $R »$, qui refuse à messieurs Kurz et Klein de le prendre entre eux, pas plus qu'il n'accepte de parcourir le monde entre madame Faul et monsieur Gefrässig, mais un beau jour rencontre «Wer " qui le prend dans ses bras, et leur rencontre fait miracle! ${ }^{24}$. Mais lorsque « Maus » et « Hund » échangent leurs têtes, leurs aventures ne font que 
commencer ${ }^{25} \ldots$ Ici, le conte a comme prétexte le langage, le lecteur est invité à participer au jeu, on lui propose des charades, des devinettes, à lui d'inventer d'autres jeux et d'autres histoires. Une fois de plus, Fühmann s'adresse à la créativité de ses lecteurs, à leur imagination, mais en même temps, si l'on considère à quel point ce livre - une des plus belles réalisations du Kinderbuchverlag par la mise en page et ses illustrations - est riche d'informations et d'enseignements, il s'adresse à leur intelligence et enrichit leur savoir. Le lecteur adulte n'est pas le dernier à se délecter à la lecture de ce livre.

16 Les éléments de merveilleux, de fantastique, d'extraordinaire sont également présents dans le reste de l'œuvre de Fühmann. On les rencontre en germe dès certains récits des années 50 - par exemple, un passage proprement surréaliste de «Le Souvenir - Rêve, 1958 ", nous montre les généraux de Hitler, dans le Bunker des derniers jours de la guerre, transformés en masques grimaçants dont le langage n'a plus rien d'humain ${ }^{26} \mathrm{ou}$ certains aspects de "Mon dernier vol ${ }^{27}$, où vision et réel se mêlent. Le recours au fantastique est particulièrement inattendu dans «Trois hommes nus ». Récit d'un réalisme dépouillé, il a pour prétexte la rencontre du narrateur avec trois hommes dans un sauna, où la nudité fait naître l'égalité, alors pourtant que les relations hiérarchiques existant entre ces trois hommes subsistent. Rencontre dans un lieu inhabituel entre le citoyen et le pouvoir, ce récit s'achève dans une dimension qui n'est plus celle du réel : alors que les trois hommes, du jour au lendemain, ont cessé de se rendre au sauna, un beau jour, le hasard les remet en présence du narrateur il les reconnait, passant dans une limousine noire :

17 Le plus grand était au volant, le plus petit à la place du passager avant et le Velu, comme au sauna, derrière eux deux : de ses yeux ronds, il scrutait le trottoir d'un air interrogateur; celui-ci se posa alors sur moi aussi et, contre toute attente, il parut vraiment me reconnaître ; il eut un léger sourire, tout à fait fugitif, bien sûr, le soupçon d'un sourire seulement mais, tout de même, d'un sourire, puis il fit un signe de tête bienveillant dans ma direction, mais déjà la voiture s'élevait et... en une ascension lente et régulière, traversa directement les airs pour entrer par une fenêtre qui, au cinquième ou sixième étage du building du marché, s'ouvrit sans bruit de l'intérieur et qui, sans bruit et légère comme une aile de papillon, se referma sur celui à qui elle venait de donner l'accès aux sphères supérieures ${ }^{28}$.

Enfin, le recours au fantastique et le dépassement du réel, éléments qui sont propres au conte, sont également présents dans les "Saiäns Fiktschen »" et dans "Vingt-deux jours ou la moitié de la vie », sous la forme de rêves intégrés au journal de voyage. Cela est également vrai dans le recueil posthume publié sous le titre de "Rêves " ${ }^{30}$, que l'on pourrait aussi intituler "Contes fantastiques"; du conte, les "Rêves» ont l'aspect «surréel » et sont en même temps, tout comme le conte, porteurs de message : très souvent, dans ces textes écrits pour certains par Fühmann peu de temps avant sa mort, ils dénoncent la guerre ou expriment, comme dans « Le rêve de l'enfant bienfaisante ", dédié en 1980 "à Stephan Hermlin pour son soixantième anniversaire», la grande lassitude de l'homme qui a lutté pour un monde meilleur où il serait enfin chez lui. Il erre sous un soleil de plomb dans une forêt immense qui semble vouloir l'engloutir, lutte sans faiblir contre le découragement, recourant comme dans le conte aux " trois souhaits » qu'il a le droit d'adresser à l'enfant bienfaisante. L'enfant, sans mot dire, le regarde avec un sourire triste et exauce son vœu: du pain et du poisson, puis une cruche d'eau. Et quand enfin - «Plutôt se tromper que ne rien faire»- le narrateur 
exprime le vœu d'être «chez lui », l'enfant surgit à nouveau devant lui et lui dit d'une voix infiniment triste : « Mais tu y es depuis le début ». Elle lui sourit avec mélancolie et disparaît : le désespoir s'empare alors de lui, il s'écroule et la forêt brûlante et muette le recouvre. Cette désespérance ne réclame guère d'exégèse...

Si le conte offre à Franz Fühmann les possibilités d'une technique narrative particulière qui lui donne un certain nombre de possibilités pour transmettre un «message », qu'il s'agisse d'habiller l'enseignement caché derrière le texte, de faire passer un message subversif - la remise en question de l'autorité en demandant «Comment le savezvous ? », le franchissement de la frontière en allant de l'autre côté du bout du monde ou d'administrer à ses lecteurs, en les divertissant, un magnifique cours de linguistique, il répond aussi, et sans doute avant tout, à l'impérieuse nécessité d'écrire ${ }^{31}$, qui est aussi celle de conter.

\section{NOTES}

1. - Zweiundzwanzig Tage oder die Hälfte des Lebens, Ges. Werke, Hinstorff Verlag, 1979.

2. - En dehors de l'œuvre citée ci-dessus, voir aussi les Essais in "Wandlung, Wahrheit, Würde », Sammlung Luchterhand 578, en particulier: Das mythische Element in der Literatur; Ernst Theodor Amadeus Hoffmann. Rede in der Akademie der Künste der DDR ; « Klein Zaches, genannt Zinnober ".

3. - In Das mythische Element in der Literatur, éd. cit., p. 62.

4. - Id., id., p. 105.

5. - Id., id., p. 65-66.

6. - Id., id., p. 66.

7. - Gespräch mit Horst Simon in Wandlung, Wahrheit, Würde, p. 255.

8. - Reineke Fuchs, Märchen nach Shakespeare, Das Nibelungenlied, Märchen auf Bestellung, Ges. Werke, Hinstorff Verlag, 1981.

9. - « Damit sind wir doch wieder beim Was-lernt-uns-das?... », op. cit., note 8, p. 320.

10. - Ein Sommernachtstraum, Id., id., p. 83-84.

11. - Id., id., p. 85.

12. - Id., id., p. 85-86.

13. - Autre exemple de la "jubilation créatrice ", ce passage du "Songe d'une nuit d'été », à propos du page de la reine Titania: «Dieses braune Kindlein war so prall und so drall und so drollig und so mollig und so verschmitzt und so gewitzt und hatte so entzückende kugelrunde Kulleraugen und tappte und trappte so tollpatschig und anmutig zugleich durch die Welt... ». Id., id., p. 87.

14. - Id., id., p. 107-108.

15. - « Die tiftlige Gnauk », ou bien l'adjectif nous suggère-t-il le venin particulier qu'elle répand par le caractère acerbe de ses réponses? Les éléments de description qui parsèment le récit permettent de se faire une idée de son aspect : «Aus dieser Beschreibung weisst du schon, Suse, dass die tiftlige Gnauke eine sehr lange Nase und sehr spitze Zähne haben muss. Ausserdem hat sie verschmitzte, sehr runde Backen, noch verschmitztere, sehr schwarze Augen und ist ausserordentlich kitzelig... Nun weisst du ungefähr, wie die tiftlige Gnauke aussieht ». Zwischen 
Erzählen und Schweigen, Franz Fühmann zum 65. Hinstorff-Verlag, 1987. Franz Marquardt, Franz Fühmann und Suse in Kötzschenbroda, p. 156.

16. - Id., id., p. 157.

17. - Id., id., p. 158.

18. - La maîtresse, dans son désarroi, s'adresse au directeur de l'école, et, de division administrative en division administrative, la question des enfants remonte jusqu'au ministre : «Die Mathelehrerin... stand vor der Tafel und runzelte die Stirne, dass die Falten über die Nase hingen, dann lief sie zum Direktor und fragte den. Der Direktor fragte den Stadtschulrat, der Stadtschulrat fragte den Bezirksschulrat, der Bezirksschulrat fragte den Republikschulrat, der Republikschulrat fragte den Minister, und der Minister sagte « Dummes Zeug », id., id., p. 158.

19. - Von der Fee, die Feuer speien konnte in : Märchen auf Bestellung, p. 291-296.

20. - Anna, genannt Humpelhexe, id., id., p. 297-307.

21. - Un exemple de ces énumérations : «Im Nu war der Wald unterm Winter begraben, und alles Leben sterbenskalt. Die Mause wollten sich schnell in die Erde wühlen, aber die war schon steinhart gefroren die Blumen erstarrten zu buntem Eis; die Hummeln fielen wie Schlössen zu Boden, und der Lerche erfroren die jubelndsten Lieder ", in Von der Fee..., p. 292.

22. - In, Anna..., p. 297.

23. - Die dampfenden Hälse der Pferde im Turm von Babel, Kinderbuchverlag, 1978. «Die Aufgabe war, Begebnisse aus den trojanischen Kämpfen zu schildern, und Hesiod sagte folgenden ersten Vers, der die Beschreibung eines Gastmahles einleiten sollte: Rindfleisch gab es zum Mahl und die dampfenden Hälse der Pferde », p. 150.

24. - Id., id., p. 72-75.

25. - Id., id., p. 79-88.

26. - «Hans hört einen Schwall von prasselnden und klappernden Lauten; bald klingt es wie Steingepolter, bald wie Eisengekreisch und bald wie Maschinengewehrtacken, so dass Hans nur einige Wörter versteht wie: frrht, vrrdg, schkslhft, gtt, ndkmpf, rrch, sndg, blschwsms, tdlchgfrr, vrrschng, thtsch, und noch einmal gttdrllmächtge... », in Erzählungen, Ges. Werke, Hinstorff Verlag, 1982, p. 104.

27. - Mein letzter Flug, id., id., p. 447.

28. - Citation française d'après : "Trois hommes nus ", in Une bagatelle en tous points positive et autres récits, éd. Alinéa Traduction, de J.-M. Argelès.

29. - Saiäns Fiktschen, Hinstorff, 1981.

30. - « Traume », publié en France dans Connaissance de la RDA, juin 1988, numéro 26.

31. - «Die andere Fragestellung, zu der einzig ich sprechen möchte, wäre die, ob Sie leben könnten, auch ohne zu schreiben, ob Sie schreiben müssen oder nicht. Dazu gibt es ein altes Wort, und das ist mein Rat für jeden Beginnenden: Ein Buch, das man nicht schreiben muss, das soll man ungeschrieben lassen ». Ein Wort an künftige Kollegen in Wandlung, Wahrheit, Würde, p. 24 .

\section{RÉSUMÉS}

Narrateur et penseur, Fühmann trouve dans le conte une des sources de son inspiration, qu'il s'agisse de puiser dans le fonds culturel universel, d'inventer de nouveaux contes ou d'étudier le conte en tant que forme narrative, établissant alors des critères permettant de distinguer entre 
le conte et le mythe.

Lorsqu'il «emprunte » des grands thèmes à la littérature universelle, Fühmann met en lumière l'actualité que présentent ces récits pour ses lecteurs, dont il sollicite réactions et réflexions.

Cette présence des lecteurs, cette attention que Fühmann leur porte est encore plus manifeste dans les contes dont la paternité lui revient complètement. Par l'irruption du fantastique dans un monde proche de celui de ses lecteurs, il déstabilise le réel dont les contradictions et insuffisances apparaissent alors de façon plus évidente.

Un des aspects de l'écriture de Fühmann conteur est qu'il utilise le langage, devenu matériau même du récit, personnage pourrait-on dire, avec une éblouissante virtuosité. Si cette virtuosité est présente de manière précoce dans son œuvre, sa manifestation la plus éclatante se trouve offerte aux lecteurs avec « Die dampfenden Hälse der Pferde im Turm von Babel».

L'importance des éléments propres au conte - dans la formulation, dans le rapport au réel, dans la place accordée au rêve, place croissante dans les textes de la maturité - est perceptible également dans le reste de l'œuvre de Fühmann. Le conte peut alors se transformer en une sorte de parabole où se reflète le réel.

Als Erzähler und als Denker findet Fühmann im Märchen eine wichtige Inspirationsquelle, sei es durch die Bearbeitung traditionneller Märchen, durch die Neuschaffung von Märchen oder indem er das Märchen als Erzählform untersucht mit dem Ziel, die prägnanten Unterschiede zwischen Märchen und Mythos festzulegen.

Wenn er sich der althergebrachten Themen der Weltliteratur bedient, hebt Fühmann ihre Aktualität für seine Leser hervor, die er zum Mitdenken und Reagieren auffordert.

Noch deutlicher werden die Aufmerksamkeit, die Fühmann seinen Lesern schenkt, ihr Einbezogensein in das Erzählgefüge, wenn Fühmann seine Märchen selbst erfindet. Der Zusammenstoss des Fantastischen mit einer Welt, die der seiner Leser ähnelt, führt die Fragwürdigkeit und Brüchigkeit der Wirklichkeit ans Licht, deren Widersprüche und Mängel deutlicher zutage treten.

Als Erzähler benutzt eigentlich Fühmann die Sprache als Element der Erzählung, beinahe möchte man sagen als Person der Handlung, und dies mit äusserster Virtuosität. Diese Virtuosität, die bereits in seinem Frühwerk auffällt, findet ihren vollkommensten Ausdruck in: « Die dampfenden Hälse der Pferde im Turm von Babel ».

Man kann die Bedeutung, die dem Märchen zukommt - sowohl in der Formulierung, als auch in der im Spätwerk immer grösser werdenden Rolle, die der Traum spielt - auch im übrigen Werk von Franz Fühmann verfolgen. Da werden oft das Märchen bzw. die Elemente aus dem Märchen zur Parabel, die die Wirklichkeit reflektiert.

\section{AUTEUR}

\section{FRANÇOISE BARTHÉLÉMY-TORAILLE}

Université de Paris X 